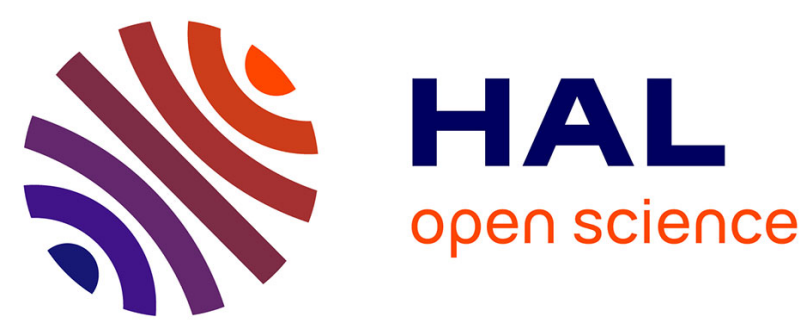

\title{
A Foot in Both Worlds: Asian Americans' Perceptions of Asian, White, and Racially Ambiguous Faces
}

\author{
Eve C. Willadsen-Jensen, Tiffany A. Ito
}

\section{To cite this version:}

Eve C. Willadsen-Jensen, Tiffany A. Ito. A Foot in Both Worlds: Asian Americans' Perceptions of Asian, White, and Racially Ambiguous Faces. Group Processes and Intergroup Relations, 2008, 11 (2), pp.182-200. 10.1177/1368430207088037 . hal-00571683

\section{HAL Id: hal-00571683 https://hal.science/hal-00571683}

Submitted on 1 Mar 2011

HAL is a multi-disciplinary open access archive for the deposit and dissemination of scientific research documents, whether they are published or not. The documents may come from teaching and research institutions in France or abroad, or from public or private research centers.
L'archive ouverte pluridisciplinaire HAL, est destinée au dépôt et à la diffusion de documents scientifiques de niveau recherche, publiés ou non, émanant des établissements d'enseignement et de recherche français ou étrangers, des laboratoires publics ou privés. 


\title{
A Foot in Both Worlds: Asian Americans' Perceptions of Asian, White, and Racially Ambiguous Faces
}

\author{
Eve C. Willadsen-Jensen and Tiffany A. Ito \\ University of Colorado at Boulder
}

\begin{abstract}
Past research on racial perception has often focused on responses from White participants, making it difficult to determine the role of perceiver race in the perception of others. Similarly, studies examining perceptions of individuals whose racial category membership is unclear have not systematically examined responses from non-Whites. This was addressed by showing Asian participants pictures of Whites, Asians, and racially ambiguous White-Asian faces. Event-related potentials were recorded to measure early attention responses. Participants initially oriented more to outgroup White than ingroup Asian or racially ambiguous faces. Shortly after that, they showed sensitivity to the racial context in which the faces were presented, more deeply processing ingroup Asian and racially ambiguous faces when they were seeing lots of other Asians, but more deeply processing outgroup White and racially ambiguous faces when they were seeing lots of other Whites. Still later, responses were more sensitive to the objective physical properties of the faces, with racially ambiguous faces differentiated from both Whites and Asians. These results demonstrate the fluidity of racial processing, and when compared to responses obtained from White participants, show how perceiver race and racial context influences attention to racial cues.
\end{abstract}

KEYWORDS context effects, ERPs, racial ambiguity, racial perception

SEVERAL influential models of person perception suggest that perceivers automatically attend to race when encountering new individuals (Bodenhausen \& Macrae, 1998; Brewer, 1988; Fiske \& Neuberg, 1990). This has been supported by recent empirical evidence (Ito \& Urland, 2003, 2005; Karylowski, Motes, Curry, \& Van Liempd, 2002; Stangor, Lynch, Duan, \& Glass, 1992), but because these studies have used primarily White samples, little is known about how the racial identity of the perceiver might influence this process. It may be reasonable to assume that all perceivers very quickly process race information, but because individuals often

\footnotetext{
$\overline{\text { Author's note }}$

Address correspondence to Tiffany A.

Ito, Department of Psychology, 345 UCB,

University of Colorado, Boulder,

CO, 80309-0345, USA

[email: tiffany.ito@colorado.edu]
} 
have more experience interacting with and perceiving members of their racial ingroup, the way in which race is processed may be affected by the race of the perceiver (Goldstein \& Chance, 1980; Rhodes, Brennan, \& Carey, 1987; Valentine, 1991; Valentine \& Bruce, 1986).

Consistent with this expectation, differences as a function of perceiver race have been obtained with respect to memory. White perceivers are generally better at remembering other Whites as compared to members of other racial groups (e.g. Buckhout \& Regan, 1988; Malpass \& Kravitz, 1969; Shapiro \& Penrod, 1986). This has been assumed to reflect an ingroup/outgroup effect in which memory is better for racial ingroup members, perhaps due to greater familiarity with the ingroup (Chance \& Goldstein, 1981; Chiroro \& Valentine, 1995). Consistent with this, Valentine and Endo (1992) found that both White (British) and Japanese faces were recognized better by ingroup than outgroup perceivers (see also $\mathrm{Ng} \&$ Lindsay, 1994). If this pattern holds more generally during person perception, we would expect responses to a particular racial group to differ depending on the race of the perceiver, producing an ingroup/outgroup effect on responses.

At the same time, there are other indications that target race exerts an influence over person perception, regardless of perceiver race. This can be seen in the same Valentine and Endo (1992) study in which the effect of perceiver race was obtained. They also found a target race main effect, with better memory for the White-British faces. Thus, while the White-British and Japanese participants were better at recognizing their own race than were participants of the other race, the Japanese participants were also fairly accurate at recognizing White faces. In fact, they were better at recognizing White-British faces than the White-British participants were at recognizing Japanese faces. Valentine and Endo suggest the Japanese participants had a high level of experience with Western culture through the media, resulting in a relatively high level of familiarity with White faces that in turn facilitated memory for members of that group. Consistent with this conclusion, research on eyewitness accounts has found that
Black eyewitnesses are more accurate when describing and identifying Whites than Whites are at identifying Blacks (Anthony, Cooper, \& Mullen, 1992). For minorities in the United States, such asymmetry is presumably a result of having a high degree of experience with the larger White community.

While memory is a domain in which effects of perceiver race have been examined, research on many other aspects of person perception has focused primarily on White participants. As a result, it has not been possible to determine whether other aspects of person perception are influenced by perceiver race or are influenced more by the race of the target group. In the present study, we sought to expand our understanding of the role of perceiver and target race by collecting responses from a sample of Asian participants. We were particularly interested in examining earlier aspects of perception to add to existing work on perceiver race and memory. To do so, we used a paradigm that we have previously used to examine relatively early perceptual responses from White participants to Whites, Asians, and Blacks (Willadsen-Jensen \& Ito, 2006), thereby allowing us to compare the responses obtained here with Asian participants to those obtained from Whites.

\section{The role of context}

It is also possible that the responses of nonWhite perceivers are not characterized by either a simple perceiver or target. Instead, the social context in which racial perception occurs may influence responses. This possibility is suggested by the fact that White perceivers may frequently have the experience of being in a primarily White environment (e.g. in their jobs, classrooms, and home life). By contrast, non-Whites may be more likely to experience different contexts, such as being among a large number of Whites at work or school, but more non-Whites at home or with friends. Such experiences may make the perceptions of non-Whites more fluid, depending on whether Whites or their racial ingroup are in the majority. Consistent with this, the effect of context in the form of solo status (being the only member of one's racial 
ingroup) was recently shown to affect the racial identity of African Americans but not of Whites (Sekaquaptewa, Waldman, \& Thompson, 2007). We have also examined context effects on White perceivers, but this question has not been addressed with non-Whites. In these studies with White participants, no effect of context on early racial perception has been observed (Ito \& Urland, 2003, 2005; Willadsen-Jensen \& Ito, 2006), but because of the contact differences just discussed, we think non-White participants might be more sensitive to racial context effects. This will be examined in the present study.

\section{Perception of racially ambiguous faces}

The present study had one final goal, that of understanding responses to individuals whose race is more ambiguous. Although race is commonly conceptualized as categorical in nature-that is, a person is perceived to be Asian or White-racial cues can and do vary continuously. An individual, for example, could be multiracial and claim membership in multiple racial categories. How individuals whose race is more ambiguous are perceived has recently received greater empirical attention (e.g. Corneille, Huart, Becquart, Brédart, 2004; Eberhardt, Dasgupta, \& Banaszynski, 2003; MacLin \& Malpass, 2001; Willadsen-Jensen \& Ito, 2006), but it is not yet known how responses to such individuals are influenced by the race of the perceiver.

\section{Present study and predictions}

The effects of perceiver and target race on racial perception, role of social context, and perceptions of racially ambiguous individuals were examined by collecting responses to White, Asian, and racially ambiguous White-Asian faces from Asian participants. In order to examine whether non-White perceivers process race differently depending on the social context, participants completed two blocks of trials that differed in overall racial composition. In one block of trials, the majority (about $87 \%$ ) of the faces seen were White and in the other, the majority were Asian.

We wished to examine both self-reported, explicit racial categorizations of the faces, as well as earlier perceptual responses. The latter were measured with event-related brain potentials (ERPs). ERPs reflect electrical brain activity in response to a discrete event, in this case, viewing of a face. Multiple components, or portions of the waveform, can be analyzed in response to the same stimulus, with different components reflecting different psychological processes. Quantifying these components allows us to examine early aspects of racial perception, and the influence of our variables of interest on relatively quick responses.

\section{P200 and N200}

Past studies have shown that several ERP components are sensitive to racial perception. Race effects have been found in an ERP component occurring within the first $200 \mathrm{~ms}$ after face onset, called the P200. This component has been associated with selective attention, with larger amplitudes associated with greater attention (Hillyard \& Munte, 1984; Luck \& Hillyard, 1994; Ritter, Simson, \& Vaughan, 1983; Wijers, Mulder, Okita, Mulder, \& Scheffers, 1989). Studies of race perception have consistently found greater attention by White participants directed to outgroup Asian and Black faces as compared to ingroup White faces (Ito, Thompson, \& Cacioppo, 2004; Ito \& Urland, 2003, 2005; Kubota \& Ito, 2007; WilladsenJensen \& Ito, 2006). Based on the direction of this effect, and on other studies finding larger P200s to more negative stimuli (e.g. angry as compared to neutral faces; Eimer \& Holmes, 2002; Eimer, Holmes, \& McGlone, 2003), we have previously suggested that race effects reflect a form of coarse vigilance. This could reflect greater attention to outgroup members either because they are more novel, or because they are perceived as more negative than ingroup members. The relatively early timecourse of the effect is consistent with a vigilance effect, which would be expected to occur early and in a fairly obligatory manner. 
Race effects have also been seen in a slightly later component, called the N200, at about $230 \mathrm{~ms}$ after face onset. Like the P200, this component has also been associated with selective attention (Hillyard \& Munte, 1984; Luck \& Hillyard, 1994; Ritter et al., 1983; Wijers et al., 1989). Here, White participants have shown greater attention to ingroup White as compared to outgroup Asian and Black faces (Ito et al., 2004; Ito \& Urland, 2003, 2005; Kubota \& Ito, 2007; Willadsen-Jensen \& Ito, 2006). The different direction of effects in the N200 as compared to the P200 suggests a different psychological mechanism, and consistent with this, N200 amplitude has been associated with depth of processing across several different contexts. For instance, larger N200s have been obtained to pictures of oneself as compared to a stranger's face (Tanaka, Curran, Porterfield, \& Collins, 2006), and to famous as compared to unfamiliar faces (Bentin $\&$ Deouell, 2000). In the case of race, the larger N200s to ingroup members is consistent with general assumptions that perceivers more deeply process ingroup than outgroup members (e.g. Brewer, 1988).

As can be seen, the P200 and N200 responses provide information on the direction of attention to members of different racial groups at different points in processing. The same responses to White and Asian faces will now be examined for Asian participants. If these responses reflect target group effects, we would expect the exact same pattern of results from Asian participants (i.e. larger P200s to Asians and larger N200s to Whites). However, if racial perception is influenced by the race of the perceiver, results should vary as a function of the ingroup/outgroup relation between the perceiver and target. In this case, Asian participants would be expected to produce a pattern of results that is the inverse of those obtained with Whites (i.e. larger P200s to Whites and larger N200s to Asians). Given that past studies on racial and non-racial perception suggest these components are sensitive to different psychological processes, it is also possible that perceiver and target group effects might differ within the two components.

\section{N400}

We also examined the $\mathrm{N} 400$, a negative-going component occurring approximately $350 \mathrm{~ms}$ after stimulus onset. We have not typically assessed the N400 in our previous studies of race perception, but in other studies of face perception, the N400 is larger to familiar than unfamiliar faces (Bentin \& Deouell, 2000; Eimer, 2000). To the degree that perceivers often have more experience with ingroup than outgroup faces, the N400 may be sensitive to the ingroup/outgroup status of the face being viewed. In this case, we would expect larger N400s to the ingroup.

\section{Late positive potential}

Finally, we examined responses at the late positive potential (LPP), ${ }^{1}$ a positive going potential that occurs around $500 \mathrm{~ms}$ after stimulus onset. The LPP is sensitive to incongruities between a target stimulus and stimuli that precede it (e.g. Cacioppo, Crites, Berntson, \& Coles, 1993; Donchin, 1981; Ito, Larsen, Smith, \& Cacioppo, 1998), with LPP amplitude increasing as a function of the difference between the two. The manipulation of racial context will create a situation in which faces are either of the same or different race as preceding faces. We expect LPP amplitude to be sensitive to this relationship, with larger LPPs to faces that are from a racial group different than the context faces.

The response to the racially ambiguous faces in each of these components is also of interest. In past studies using White perceivers, the responses to racially ambiguous Asian-White and Black-White faces were indistinguishable from those to ingroup White faces at the P200 and N200 (Willadsen-Jensen \& Ito, 2006). We interpreted this pattern as indicating that early processing is more gross than fine-grained, with no distinction made between a $100 \%$ and $50 \%$ ingroup face within the first $250 \mathrm{~ms}$. It was not until later in processing, in both the LPP and in self-reported racial categorizations, that the racially ambiguous faces differed from both ingroup and outgroup faces. In the present study, if responses in earlier ERP components reflect a target group effect, Asian participants should 
show the same responses to racially ambiguous faces that White participants have. In this case, the racially ambiguous faces should elicit the same P200 and N200 responses as White faces. However, if the similar P200 and N200 responses elicited by White and racially ambiguous faces is related to White faces being the ingroup, we would expect the inverse pattern from our Asian participants. In this case, the racially ambiguous faces should elicit the same responses as ingroup Asian faces. In our past studies, responses to racially ambiguous faces have differed from those to both ingroup and outgroup faces later in processing, as reflected in the LPP ERP component and self-reported responses. We expect to replicate this here.

\section{Method}

\section{Participants}

Twenty-one Asian undergraduate students (12 males) participated in this study; participants were either paid or received partial class credit. One male participant was dropped due to excessive eye movement, which obscured the ERP data. The majority of participants $(N=17)$ were born in the United States; all had lived in the United States for at least the immediately preceding 12 years. Self-reported interracial contact information confirms that all participants had a high degree of contact with Whites. Participants specifically reported on the number of White high school friends, childhood neighbors, and current friends (where $1=$ 'None' and $7=$ 'Many'). Mean scores across the three items was 5.78. Of interest, the mean rating for these same questions answered with respect to Asians was significantly lower, at $3.44(t(19)=6.26, p<.001)$. Thus, although our sample was Asian and presumably had intimate contact with other Asians at least through family interactions, our participants reported relatively more contact with Whites in their neighborhood and among friends.

\section{Materials}

Forty-two pictures each of White American and Asian American men and 12 racially ambiguous
White-Asian men were used as target stimuli. All were head and shoulder poses of young adults edited to have a uniform blue background and black clothing and taken from WilladsenJensen and Ito (2006). Racially ambiguous faces were created from an Asian 'parent' and a White 'parent' that were digitally morphed in equal proportions. The faces used to create the morphs were different than the ones used in the experiment. All faces were matched on attractiveness and pretested extensively for race and believability, as described in Study 1 of Willadsen-Jensen and Ito (2006).

\section{Procedure}

The design and procedure also follow from Willadsen-Jensen and Ito's (2006) first study. Participants were introduced to the procedure for affixing electrodes and provided informed consent. The experimenter then placed an elastic cap, into which the electrodes were sewn, on each participant's head (Electro-cap International, Eaton, OH). ERPs were recorded from 26 scalp locations using tin electrodes. Miniature tin electrodes were also placed above and below the left eye and next to the outer canthus of each eye to monitor vertical and horizontal eye movements. Scalp electrodes were referenced online to the left mastoid site. Once the electrodes were in place and all impedances were below $5 \mathrm{~K} \Omega$, the participants received further instructions telling them the experiment would measure their brainwaves while they looked at pictures of people. They were asked to categorize the people as White or Asian, registering their answers using an appropriately labeled keypad (order of labels was counterbalanced across participants).

All participants completed a White context and an Asian context block of trials in random order. In the White context block, participants saw sequences of four pictures of White males and a fifth 'target' picture during which data were collected that was either another White, Asian, or racially ambiguous face. In the Asian context, participants saw sequences of four pictures of Asian males and a fifth 'target' picture that was either another Asian, White, or racially 
ambiguous face. Each block consisted of 30 trials of each type, for a total of 90 trials/block, where a trial consisted of four context pictures and one target picture. The target picture randomly appeared in either the third, fourth, or fifth position within a trial. Each face was shown for $1000 \mathrm{~ms}$, separated by a $1200 \mathrm{~ms}$ inter-trial interval. Participants registered their explicit racial categorization judgment after picture offset. After completing both blocks, participants completed a demographic questionnaire then were debriefed and thanked.

\section{Psychophysiological data collection and reduction}

ERP recordings were amplified with a gain of 500 by NeuroScan Synamps model amplifiers with a bandpass of 0.1 to $30 \mathrm{~Hz}$ (12-dB roll-off) and digitized at $1,000 \mathrm{~Hz}$. Offline, the data were rereferenced to an average of the left and right mastoids, and an eye blink algorithm was used to remove the effects of vertical eye movements from the ERP, which can distort measurements from scalp sites (Semlitsch, Anderer, Schuster, \& Presslich, 1986). Data were epoched into segments beginning $128 \mathrm{~ms}$ before picture onset and continuing throughout the picture presentation. The data were then corrected to the mean voltage of the prestimulus recording period. Trials were visually inspected for remaining ocular or other artifact (e.g. due to movement); data from all sites for that trial were eliminated from further analysis if an artifact was detected. Finally, ensemble averages were constructed by aggregating the electrical activity associated with each of the three target conditions in each block for each participant at each scalp site.

\section{Results}

\section{ERP results}

The peak amplitude and latency of five potentials were scored within each participant's ensemble averages by locating the maximal negative deflections between $80-130 \mathrm{~ms}$ (N100), 200-300 ms (N200), and 200-300 ms (N400) and the maximal positive deflections between $150-250 \mathrm{~ms}$ (P200) and 300-600 ms (LPP). The N100 did not yield any results of theoretical interest; ${ }^{2}$ therefore, analyses of only the P200, N200, N400 and LPP are reported. These components have been maximal at midline scalps sites in past research, and preliminary analyses conducted on all 26 scalp sites confirmed this. For simplicity, analyses are, therefore, restricted to the midline frontal $(\mathrm{Fz}$,$) central (\mathrm{Cz})$, and parietal $(\mathrm{Pz})$ electrodes.

A separate 2 (Participant Gender: male, female) $\times 2$ (Context Race: White context, Asian context) $\times 3$ (Target Race: White, Asian, racially ambiguous) $\times 2$ (Context Order: White context first, Asian context first) $\times 3$ (Scalp Site: $\mathrm{Fz}, \mathrm{Cz}$, $\mathrm{Pz}$ ) mixed model repeated measures analysis of variance (ANOVA) was run for each ERP component. All factors except participant gender and context order were within subjects. No significant effects or interactions occurred with participant gender or context order; therefore, these factors were dropped from subsequent analyses. $^{3}$

P200 Figure 1 shows the ERP waveforms averaged across participants for White, Asian, and racially ambiguous faces. The top panel shows responses during the White Majority Context and the bottom panel shows responses during the Asian Majority Context. The P200 can be seen as the first positive-going component in Figure 1, peaking with a mean latency of $170 \mathrm{~ms}$. Although all three scalp sites were analyzed, waveforms are just shown at the $\mathrm{Cz}$ scalp site for simplicity. (Note that positive voltages are plotted downward.)

Consistent with past research, the P200 was sensitive to Target Race $(F(2,38)=6.91$, $p<.01)$. Mean P200 amplitudes are shown in Figure 2. Recall that White participants show larger P200s to Asian than White and racially ambiguous faces (Willadsen-Jensen \& Ito, 2006). As can be seen, the Asian participants showed the opposite pattern. That is, simple effects tests showed significantly larger P200s to White $(M=6.55 \mu \mathrm{V})$ than Asian $(M=5.34 \mu \mathrm{V})$ $(F(1,19)=17.06, p<.01)$, and racially ambiguous faces $(M=5.01 \mu \mathrm{V})(F(1,19)=5.26, p<.05)$. At this point in the processing, P200s did not 
A. White majority context

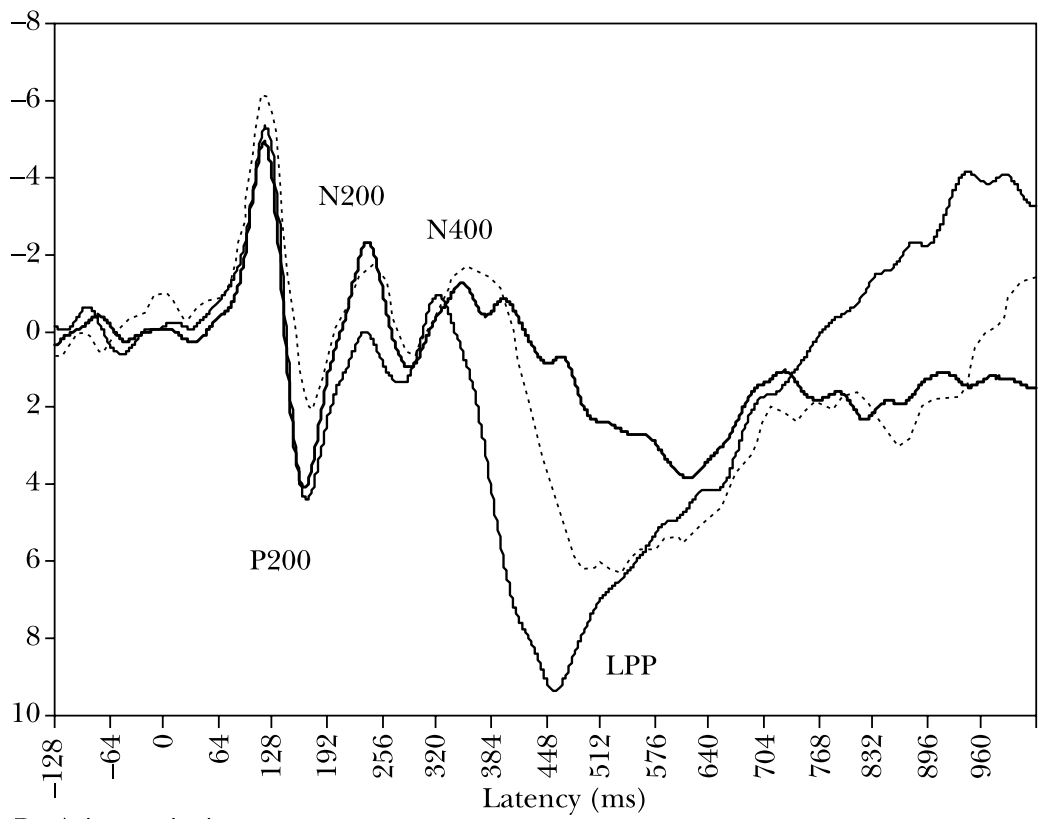

B. Asian majority context

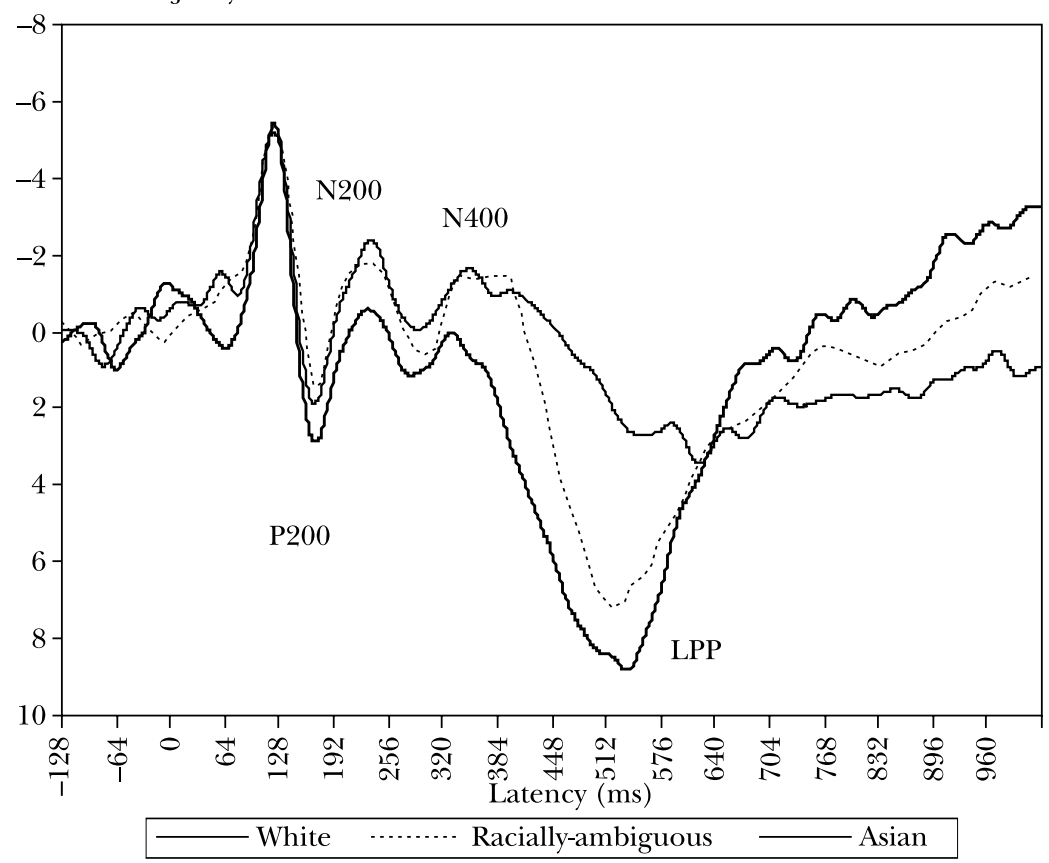

Figure 1. ERP waveforms at the $\mathrm{Cz}$ scalp site averaged across participants for White, Asian, and racially ambiguous faces. Panel A shows responses during the White Majority Context and Panel B shows responses during the Asian Majority Context. 


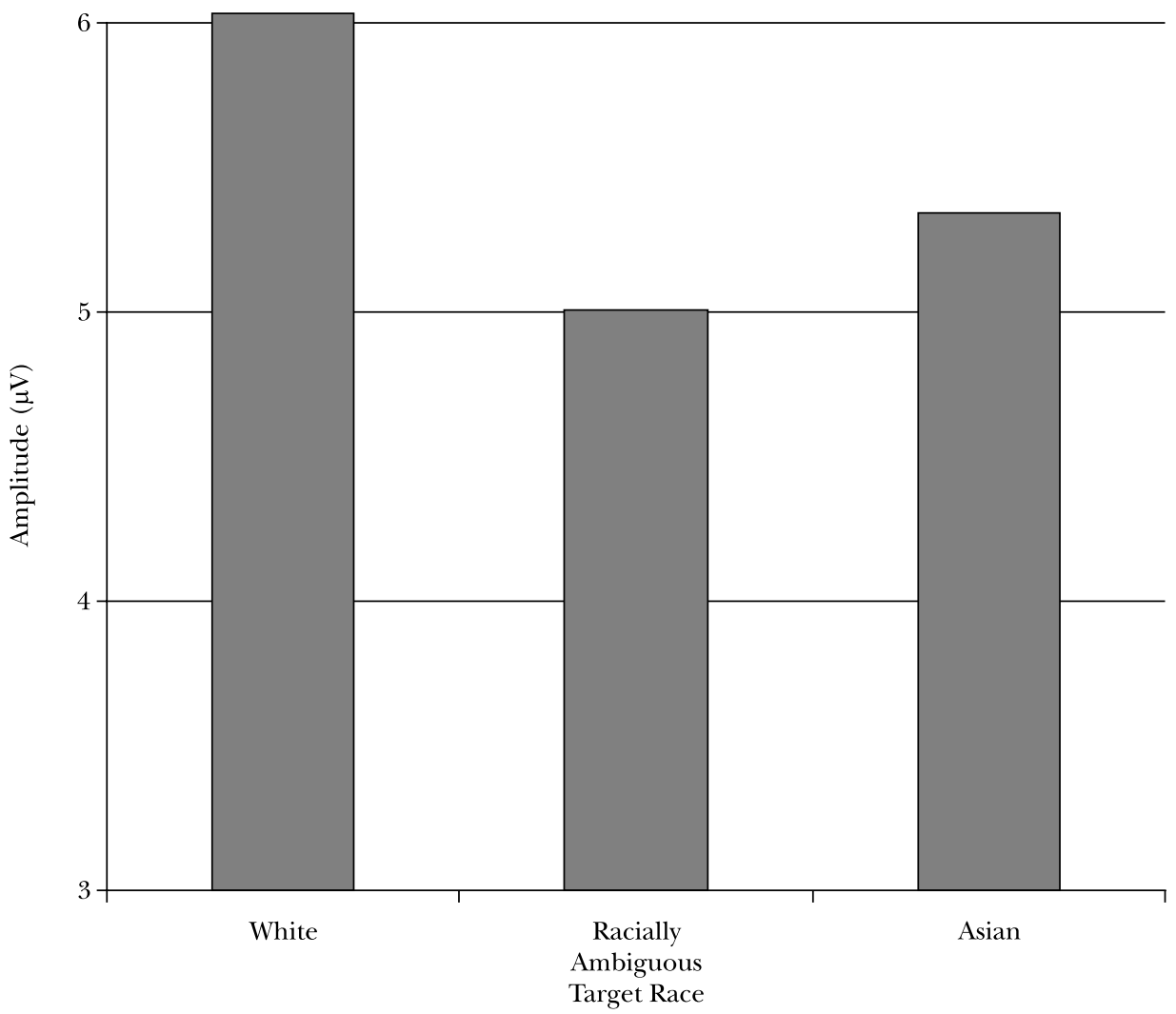

Figure 2. Mean P200 amplitude for White, Asian, and racially ambiguous faces.

differ to Asian and racially ambiguous faces $(F(1,19)=1.65, n s)$. Thus, the $\mathrm{P} 200$ displays a pattern of ingroup/outgroup processing in which perceivers initially orient more attention to unambiguous outgroup members, while no difference is seen between ingroup and racially ambiguous faces.

The only other significant effect was the main effect of Context Race showing that P200 amplitudes were larger in the White context block $(M=4.88 \mu \mathrm{V})$ than the Asian context block $(M=3.82 \mu \mathrm{V}) \quad(F(1,19)=4.84, p<.05) .{ }^{4}$ This was not expected, but suggests greater attention overall in the block in which more White than Asian or racially ambiguous faces were seen.

N200 The N200 peaked with a mean latency of $232 \mathrm{~ms}$ (see Figure 1). The main effect of Target Race was not significant $(F(2,38)=2.42$, $p=.10)$, but the Target Race $\times$ Context Race interaction was $(F(2,38)=5.51, p<.05)$. The nature of the interaction was explored by examining simple effects of Target Race within the two Context Races. In past research using White participants, N200s have been larger to White and racially ambiguous as compared to Asian faces (Willadsen-Jensen \& Ito, 2006). As seen in Figure 3, this same pattern was obtained in the White context. White $(M=-2.79 \mu \mathrm{V})$ and racially ambiguous $(M=-3.24 \mu \mathrm{V})$ faces were associated with significantly larger N200s than Asian faces $(M=-1.00 \mu \mathrm{V}) \quad\left(F_{\mathrm{s}}(1,19)=5.02\right.$ and 5.39, $p \mathrm{~s}<.05)$. As in past research, N200s to White and racially ambiguous faces did not differ $(F(1,19)=0.58, n s)$.

The opposite pattern was obtained in the Asian context block. Here, N200s were significantly larger to Asian $(M=-3.43 \mu \mathrm{V})$, and marginally 


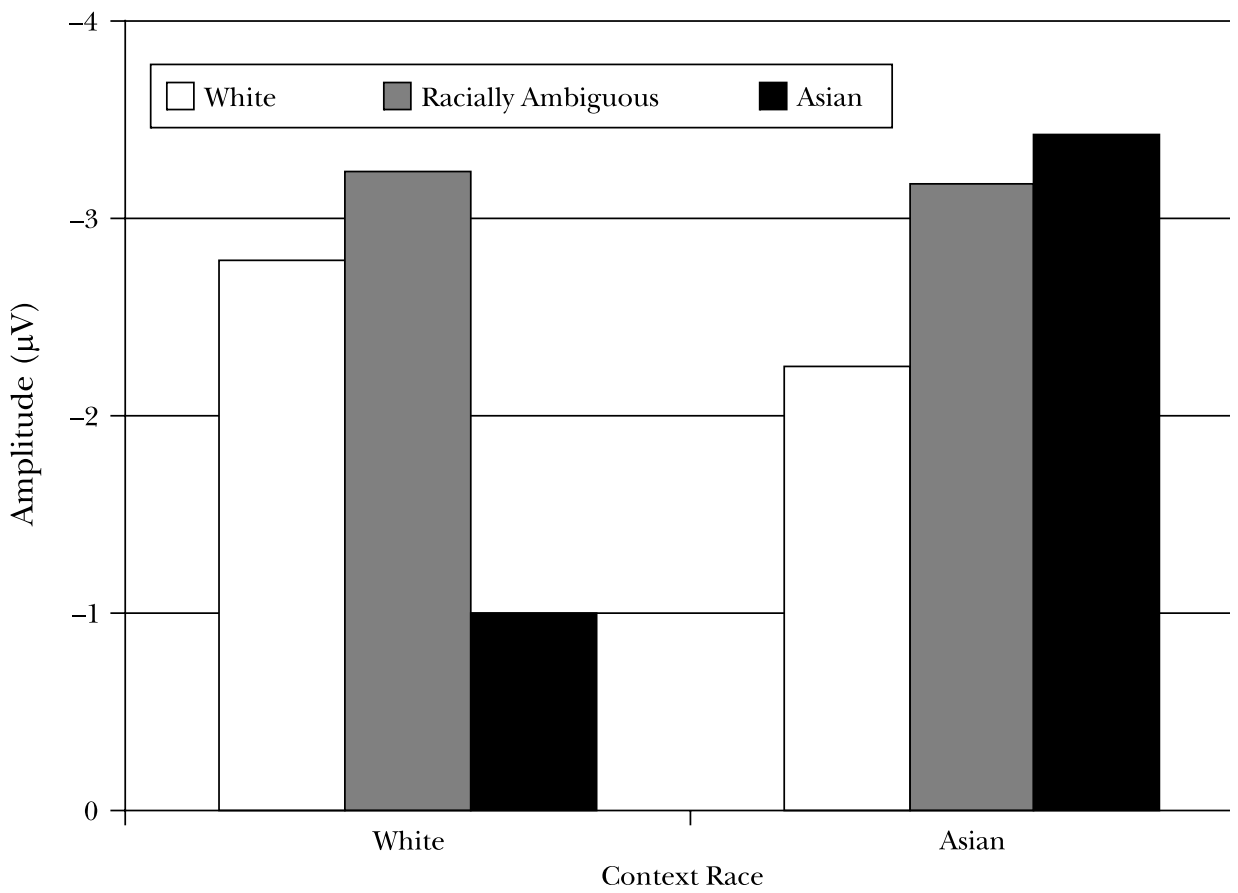

Figure 3. Mean N200 amplitude for White, Asian, and racially ambiguous faces. The left side of the graph shows responses during the White Majority Context and the right shows responses during the Asian Majority Context.

significantly larger to racially ambiguous $(M=-3.18 \mu \mathrm{V})$ than White faces $(M=-2.25 \mu \mathrm{V})$ $(F \mathrm{~s}(1,19)=4.68$ and $2.71, p<.05$ and $p=.11$, respectively). N200s were equally large to Asian and racially ambiguous faces $(F(1,19)=$ $.19, n s) .^{5}$

This pattern of results shows that the responses of Asian participants shift depending on the context in which racial perception occurs. When perception occurs in the context of primarily White faces, Asians show the same pattern obtained from White participants (i.e. larger N200s to White and racially ambiguous faces). However, when perception occurs in the context of primarily ingroup Asian faces, they now show greater attention to Asian and racially ambiguous faces.

N400 We refer to the next negative-going component, peaking with a mean latency of $375 \mathrm{~ms}$, as the N400 (see Figure 1). Analyses revealed a
Target Race main effect $(F(2,38)=4.80, p<.05)$. $\mathrm{N} 400$ s tended to be larger to racially ambiguous and Asian than White faces. Specifically, $\mathrm{N} 400$ s were significantly larger to racially ambiguous $(M=-2.43 \mu \mathrm{V})$ than White faces $(M=-0.93 \mu \mathrm{V})(F(1,19)=9.80, p<.01) . \mathrm{N} 400 \mathrm{~s}$ to Asian faces $(M=-1.86 \mu \mathrm{V})$ were marginally larger than those to Whites $(F(1,19)=3.49$, $p=.077)$, while N400s to racially ambiguous and Asian faces were equally large $(F(1,19)=$ $1.36, n \mathrm{~s})$.

This effect was marginally moderated by Context Race $(F(2,38)=3.02, p=.06)$. Looking separately within each context race, there were no significant effects of target race within the White context (see Figure 4), $F \mathrm{~s}<1$. In the Asian context block, the pattern replicates that obtained in the N200 in the same context. That is, $\mathrm{N} 400$ s were larger to Asian $(M=-2.31 \mu \mathrm{V})$ and racially ambiguous $(M=-2.98 \mu \mathrm{V})$ than White faces $(M=-0.36 \mu \mathrm{V})(F(1,19)=8.83$ and 38.48, 


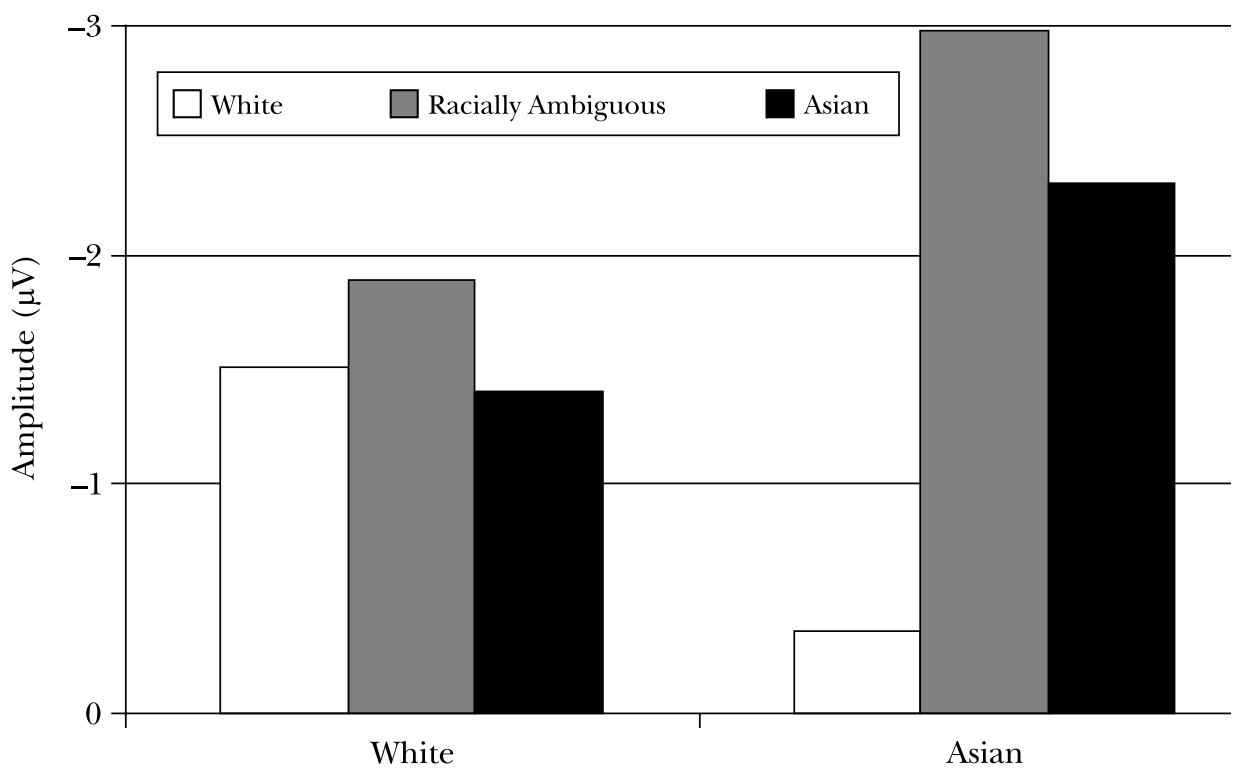

Context Race

Figure 4. Mean N400 amplitude for White, Asian, and racially ambiguous faces. The left side of the graph shows responses during the White Majority Context and the right shows responses during the Asian Majority Context.

$p \mathrm{~s}<.001)$. N400s did not differ to the Asian and racially ambiguous faces $(F(1,19)=1.05, n \mathrm{~s})$. Thus, like the N200, the N400 provides some indication of a context effect in which greater attention is directed to Asian and racially ambiguous faces, but only when perception occurs in the context of primarily ingroup Asian faces. ${ }^{6}$

LPP The final component of interest, the LPP, peaked with a latency of $508 \mathrm{~ms}$ (see Figure 1). LPP amplitude is typically determined by the relationship between a target stimulus and the stimuli that precede it, with LPP amplitude increasing as a function of the difference between the two (e.g. Cacioppo et al., 1993; Donchin, 1981; Ito et al., 1998). In the current study, where ERPs were recorded to faces that were viewed within the context of either White or Asian faces, we expect LPP amplitude to vary as a function of the match between the race of the context and target faces. Consistent with this, we obtained a significant Target Race $\times$ Context Race interaction $(F(2,38)=38.89, p<.0001)$. Mean LPP amplitudes are shown in Figure 5. Simple effects tests within each context race block revealed the expected racial incongruity effect in the form of a graded pattern that reflected the objective composition of the faces. That is, within each context, LPPs were smallest to the faces that were of the same race as the context, next largest to the racially ambiguous faces, and larger still to the racially unambiguous faces that differed from the context. Thus, in the White context block, LPPs were significantly larger to the contextually incongruent Asian $(M=10.68 \mu \mathrm{V})$ than congruent White faces $(M=3.78 \mu \mathrm{V})$ $(F(1,19)=55.80, p<.0001)$. Similarly, within the Asian context block, LPPs were significantly larger to contextually incongruent White $(M=10.27 \mu \mathrm{V})$ than congruent Asian faces $(M=3.65 \mu \mathrm{V})(F(1,19)=50.39, p<.0001)$. In both contexts, LPPs to the racially ambiguous faces were intermediate to the responses to the Whites and Asians, reflecting their composition as $50 \%-50 \%$ blends of White and Asian faces. In the White context block, LPPs 


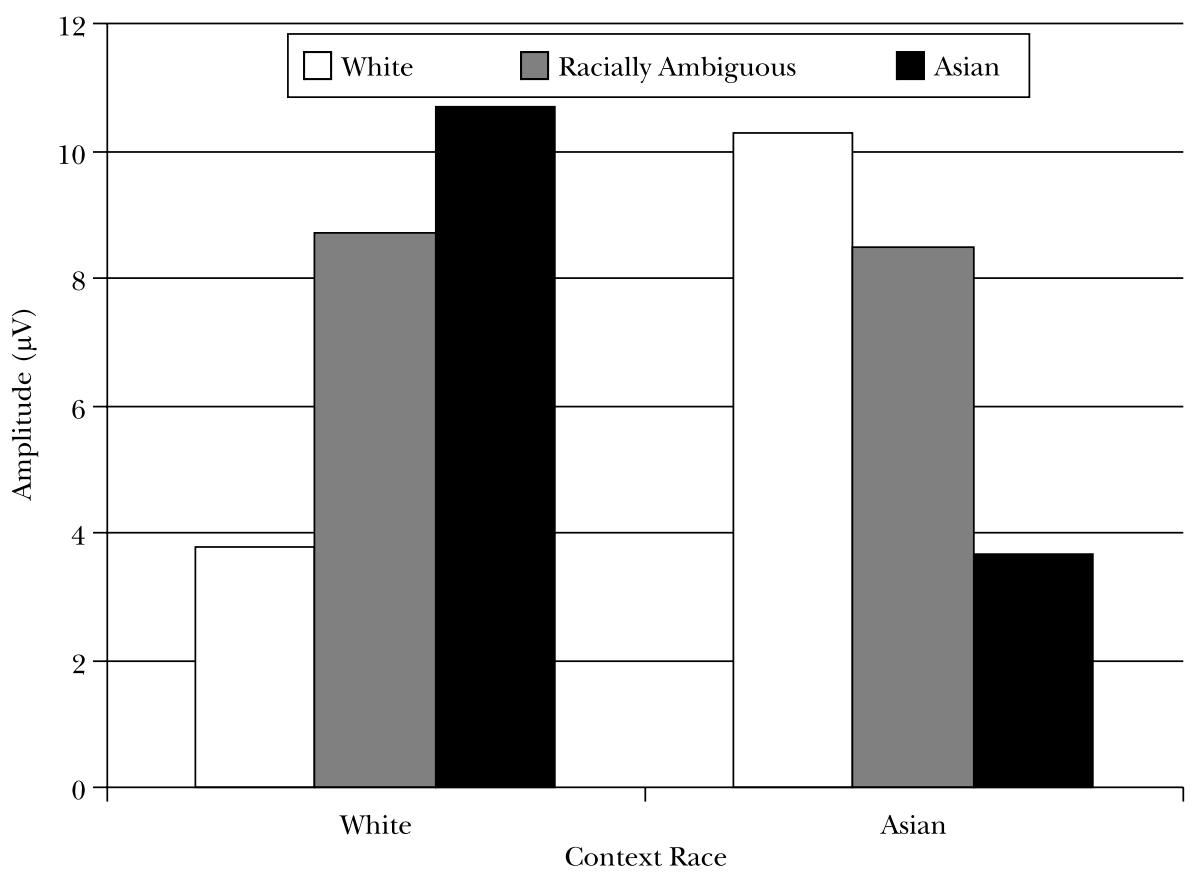

Figure 5. Mean LPP amplitude for White, Asian, and racially ambiguous faces. The left side of the graph shows responses during the White Majority Context and the right shows responses during the Asian Majority Context.

to racially ambiguous faces $(M=8.71 \mu \mathrm{V})$ were significantly larger than those to contextually congruent Whites $(F(1,19)=29.00 p \mathrm{~s}<.001)$, but significantly smaller than those to Asians $(F(1,19)=6.91, p<.05)$. Similarly, in the Asian context block, LPPs to racially ambiguous faces $(M=8.48 \mu \mathrm{V})$ were significantly larger than those to contextually congruent Asians $(F(1,19)=15.25, p<.001)$, but significantly smaller than those to Whites $F(1,19)=4.53$, $p<.05) .^{7}$

\section{Explicit categorization}

Nearly $100 \%$ agreement occurred in categorizing Asian faces as Asian and White faces as White. Analyses were therefore restricted to responses to the racially ambiguous faces. Overall, the racially ambiguous faces were perceived as ambiguous; across blocks, the percentage of times a racially ambiguous face was categorized as Asian was $51 \%$.

For analysis, participants' dichotomous race choice was represented as the percentage of times racially ambiguous faces were categorized as Asian. These were then analyzed in a 2 (Participant Gender: male, female $) \times 2$ (Context Race: White context, Asian context) $\times 2$ (Context Order: White context first, Asian context first) repeated measures mixed-model ANOVA. ${ }^{8}$ Gender did not yield any significant effects and was dropped from the analysis. The only significant effect was the Context Race main effect, indicating a contrast effect $(F(1,15)=6.80, p<.05)$. Racially ambiguous faces were categorized as Asian more frequently when seen in the context of primarily White $(M=74 \%)$ than Asian faces $(M=43 \%)$. Both means are significantly different from $50 \%: \chi^{2}(16)=138.47$ and $95.74, p<.001$, respectively.

\section{Discussion}

The current study was designed to expand our understanding of racial perception by more systematically analyzing responses from nonWhite participants. This allowed us to examine 
whether early aspects of racial perception differ as a function of perceiver race, or are primarily determined by target race. To do so, we recorded responses from Asian participants and compared their responses to those obtained in past research from White participants (Willadsen-Jensen \& Ito, 2006). Looking across the two studies provides information in all four cells of a crossover design in which participants of two different races respond to targets of both races. Our design also allowed us to examine the effects of social context on race perception.

\section{Responses to White and Asian faces}

Results indicate that racial perception is sensitive to several factors, including perceiver race, target race, and target context, and that the influence of these factor differs at different points in time. Considering the responses to just the White and Asian faces first, clear evidence for the effect of perceiver race was seen in the P200. Asian participants in the present study showed larger P200s to outgroup White than Asian faces. When viewed in the context of past results from White participants, who show larger P200s to outgroup Asian and Black than White faces (Ito \& Urland, 2003, 2005; Kubota \& Ito, 2007; WilladsenJensen \& Ito, 2006), these results clearly show greater attention to outgroup faces across perceivers and targets of different races. As noted in the introduction, past findings that the P200 is larger to racial outgroup than ingroup faces (Ito et al., 2004; Ito \& Urland, 2003, 2005; Kubota \& Ito, 2007; Willadsen-Jensen \& Ito, 2006) and to faces displaying negative as compared to neutral expression (Eimer \& Holmes, 2002; Eimer et al, 2003) has lead to suggestions that the P200 may index relatively early vigilance processing (Ito \& Urland, 2003; Kubota \& Ito, 2007). Finding that Asian participants similarly show larger P200s to White targets further supports this interpretation. Moreover, the findings allow us to generalize the conclusion that race is processed quickly (and probably with little effort) beyond just White perceivers (see also, Dickter \& Bartholow, 2007).

But rather than an inflexible pattern of ingroup/outgroup processing throughout person perception, the results also indicate that the racial perception of Asians is affected by the racial context in which a person is encountered. For White participants, we have found larger N200s to Whites than Asians or Blacks (Ito et al., 2004; Ito \& Urland, 2003, 2005; Kubota \& Ito, 2007; Willadsen-Jensen \& Ito, 2006). If this reflects an ingroup/outgroup pattern of responding, we would expect the Asian participants in the present study to show larger N200s to ingroup Asians than outgroup Whites. This pattern was obtained, but only when the faces were seen in the context of primarily Asian faces. By contrast, when Asian participants were viewing primarily White faces, they showed the same pattern as White participants; N200s were larger to White than Asian faces.

Past research on face perception has associated N200 amplitude with deeper processing (Bentin \& Deouell, 2000; Tanaka et al., 2006). If the N200 we have obtained in the context of racial perception similarly reflects depth of processing, results from White participants indicate a tendency for deeper processing of ingroup White faces, consistent with other findings indicating preferential attention to ingroup members (e.g. Brewer, 1988; Levin, 1996, 2000). The context effects seen in the N200 in the present study, however, suggest that Asian perceivers do not simply more deeply process ingroup members in all situations. Instead, they flexibly attend to individuals from whatever the most numerous racial group is at the time.

It is as if Asian perceivers can adopt a perspective that matches that of White perceivers when they find themselves in a context that involves primarily White individuals. This is consistent with the idea that non-Whites in the United States must learn to navigate two social realms: their own and that of the White majority. In support of this, the tendency for better recognition of racial ingroup than outgroup members is very robust in White Americans, but weaker and more inconsistent in African Americans (Anthony et al., 1992; Feinman \& Entwisle, 1976; Golby, Gabrieli, Chiao, \& Eberhardt, 2001), presumably because the latter have a high degree of experience interacting with outgroup Whites. As would be predicted from the memory research, past studies employing the same 
context manipulation with White participants have failed to reveal context effects in the P200 or N200 (Ito \& Urland, 2003, 2005; WilladsenJensen \& Ito, 2006). This suggests that context alone is not sufficient to alter perceptions; it has effects only in certain participants. That our Asian participants would be facile at adopting different racial perspectives is supported by the high rate of contact they report with Whites. In fact, our participants reported more contact among high school friends, childhood neighbors, and current friends with outgroup Whites than ingroup Asians.

The effect of context on N200 responses occurred in response to a relatively subtle manipulation. The numerical composition of the faces differed, but we did not implement any specific contingencies based on these differences. Although the effect of context on racial perception has not been widely investigated, studies that have focused on the issue tend to examine the influence of longer-term context effects. For instance, Sangrigoli and colleagues recently showed the typical own-race memory advantage with Korean and French Caucasian participants (Sangrigoli, Pallier, Argenti, Ventureyra, \& de Schonen, 2005). Of relevance to the present results, adults of Korean origin who were adopted into Francophone Caucasian families when they were between 3 and 9 years of age showed better memory for outgroup Caucasian than ingroup Korean faces. An own-race recognition advantage has been observed with children as young 3 months (Sangrigoli \& de Schonen, 2004, see also Pezdek, Blandon-Gitlin, \& Moore, 2003), well before the point at which the Korean children were adopted into Caucasian families. From this, Sangrigoli et al. assumed that the early life experiences of the Korean adoptees initially afforded them better expertise in recognizing ingroup Korean faces. That this advantage disappeared by adulthood demonstrates the long-term contextual influence of living among primarily Caucasians (see also, Bar-Haim, Ziv, Lamy, \& Hodes, 2006). The present results expand on these findings by showing context effects in another domain (that of early racial perception) and that effects can be obtained with only short-term manipulations. Moreover, the present effects manifested very quickly in perception. The N200 peaked with a latency of $232 \mathrm{~ms}$, indicating that differences in depth of processing which were sensitive to racial context likely occurred fairly implicitly as opposed to after explicit strategic consideration.

Offering converging evidence that early aspects of racial perception are affected by perceiver race, Dickter and Bartholow (2007) have recently reported that both White and Black participants show larger P200s to outgroup faces but larger N200s to ingroup faces. We, therefore, obtain the same pattern in the P200, but not the N200. Our evidence of a context effect in the N200 likely reflects differences in procedure, as Dickter and Bartholow did not employ a racial context manipulation. Together, the results from the two studies suggest that perceivers may typically differentiate between ingroup and outgroup members, but that nonWhite perceivers might also have the ability to respond in the same way that White perceivers do when they find themselves in a primarily White racial context.

The N400 has differentiated between familiar and unfamiliar faces (Bentin \& Deouell, 2000; Eimer, 2000). Because individuals often have greater familiarity with ingroup than outgroup members, we might expect larger N400s to ingroup Asian faces in the present study. This was obtained when faces were seen in the context of primarily Asians, but when faces were seen in the context of primarily Whites, N400s did not differ to Whites and Asians. The lack of differences in the White context may be related to the effects of racial context obtained in the N200. Any tendency for familiarity with the ingroup to increase N400s to Asian faces may have been diluted by the majority of White faces in the White context block.

\section{Responses to racially ambiguous faces}

In addition to considering how racial group membership and context influences the perception of individuals whose race can be easily determined, we were also interested in exploring how Asian perceivers view racially ambiguous White-Asian faces. In studies involving White participants, we find that reactions to racially ambiguous 
faces are initially undifferentiated from those to ingroup White faces (Willadsen-Jensen \& Ito, 2006). We have suggested that this reflects the coarseness of initial aspects of face processing. The racially ambiguous faces are a $50 \%-50 \%$ blend between two races, and if the type of processing that occurs within the first $200-300 \mathrm{~ms}$ after the onset of the face is less refined, differences between a $100 \%$ ingroup and $50 \%$ ingroup face may not be appreciated yet.

Replicating these past results, racially ambiguous faces elicited the same $\mathrm{P} 200$ response as ingroup Asian faces. In the N200 and N400, where context affected responses to the White and Asian faces, the pattern of differences in comparison to the racially ambiguous also changed with context. In the Asian context block, where N200s were larger to ingroup Asians than outgroup Whites, N200s were equally large to Asian and racially ambiguous faces. By contrast, in the White context block, where N200s were largest to outgroup White faces, N200s were equally large to White and racially ambiguous faces. Similarly, in the N400 in the Asian context block, N400s were equally large to ingroup Asian and the racially ambiguous faces. In the White context block, where N400s did not differ to the White and Asian faces, the racially ambiguous faces elicited responses that were indistinguishable from either. To the degree that Asian perceivers are able to shift their mode of processing to direct more attention to either Whites or Asians depending on context, the similarity in responses between the racially ambiguous faces and Asian as compared to White faces similarly changed. Context appeared to change the reference group for Asian perceivers. Ingroup Asians are normally the reference group, but in some situations (e.g. when lots of other Whites are being seen), Whites can become the reference group. Responses to racially ambiguous faces tend to match those of the reference group.

In our past research with White participants, although responses to the racially ambiguous faces did not differ from those to ingroup faces in the P200 and N200, they did differ from both ingroup and outgroup faces in the LPP and in self-reported categorization. Both effects were also obtained in the present study with Asian participants. This shows two things. First, although participants initially did not fully differentiate the racially ambiguous faces from Asian and White faces, the difference was appreciated as processing continued. Second, at the point in processing when the difference was appreciated, participants were fairly sensitive to the physical properties of the faces, showing a graded response in which racially ambiguous faces were responded to in a manner that was between the White and Asian faces, mirroring the objective composition of the faces.

The differentiation of the racially ambiguous faces from the Whites and Asians in self-reported categorization took the form for a contrast effects relative to the context race. This is not surprising given the ambiguity of the faces. Seeing faces from primarily one racial group may highlight the ways in which the racially ambiguous faces differ from that group. It is particularly interesting that this contrast effect only manifests in the explicit categorization responses, indicating that the type of comparative process that produced it emerged relatively late in processing, as participants were determining an explicit racial categorization. Of course, we did not give participants the option of specifying a racial group other than White or Asian. We did this primarily to simplify the categorization task, which was beneficial for the collection of the ERP data (e.g. because it allowed us to use a relatively short interstimulus interval, critical given the large number of pictures shown). It is possible that the contrast effect would be eliminated if participants were given different response options, such as a multiracial category, or even allowed to make open-ended responses. But we do not think this makes the present results uninteresting. We suspect that perceivers often approach the world using only a few racial categories. Tiger Woods, for instance, is often referred to as an African American golfer, despite actually being multiracial (he has described himself as Caucasian, Asian, African American, and Indian).

\section{Changes in racial perception across time}

One benefit of using ERPs to study social perception is the ability to measure different aspects of perception as they unfold online, over time. 
This is an advantage over both behavioral measures, and other neuroscience measures such as functional magnetic resonance imaging, which have a much slower timecourse. Although perceivers may only become aware of the explicit outcome of social perception (e.g. the categorization of what race someone is), any single decision is likely to have been supported by a cascade of stages. Past research using ERPs to study social perception suggests that different ERP components are sensitive to somewhat different factors. The present results continue to support these distinctions, but more importantly, they demonstrate that several factors influence the racial perception of Asian perceivers at these different points in time.

We can consider the implication of the ordering of the obtained effects. The P200, which may be linked with vigilance in the context of social perception, was characterized by an ingroup/outgroup pattern of responses. It seems reasonable that vigilance processes would be influenced by features of the stimulus as well as chronic aspects of the perceiver which color their interpretation of external events. Although vigilance can also be influenced by context (consider how interpretations of the same event might differ in the light of day versus dark of night), context effects in the present study would require at least a rudimentary comparison between the present individual and the social category of individuals seen most frequently in that block of trials. This would likely take longer to manifest than a response dependent on only perceiver and/or target characteristics. Consistent with this, the context effects took slightly longer to occur, manifesting in the N200 and N400 (although they still occurred fairly quickly in the absolute sense). It is worth noting that although greater attention may be initially directed at outgroup members (as reflected in the P200), there is little need for continued vigilance in the present setting. Participants are sitting alone in a lab, and there is no potential for interaction between them and the individuals they are viewing. Other factors such as context likely have more opportunity to operate when personal safety concerns are low. Lastly, longer viewing of the faces gives participants greater opportunity to appreciate the specific composition of the racially ambiguous faces. Responses that more closely reflect the 50\%-50\% White-Asian composition of the racially ambiguous faces emerge after both the ingroup/outgroup and context effects, manifesting in the LPP and explicit self-reported categorization.

\section{Implications}

These results show that racial perception is clearly influenced by the perceivers' racial group membership, a conclusion not always obvious from past research because of a lack of variability in perceiver race. While perceivers of all races seem to very quickly attend to racial information, the way in which that information affects the direction of attention differs depending on one's race. Of equal importance, the results also show that racial perception can be affected by the racial context in which an individual is encountered. We think it particularly interesting that the context effects obtained in the N200 and N400 have not been observed in data collected from White participants (Ito \& Urland, 2003, 2005; Willadsen-Jensen \& Ito, 2006). Members of minority racial groups typically need to be fluent at interacting not only with their own culture but also the majority culture. By contrast, Whites may more often have the experience of being in the presence of primarily other Whites. Non-Whites may, therefore, have a foot in two worlds, and our results suggest that racial perception can fluidly change to accommodate these different experiences.

Considering racial perception as sensitive to different factors across time suggests the possibility of manipulations that selectively effect particular stages. That was demonstrated to some extent with the context manipulation having effects on only the N200 and N400. We might expect other dissociations. For instance, manipulations that affect a sense of threat may be more likely to have effects in the P200, whereas individuation manipulations might have their effects later, in the N200.

We were not able to measure other aspects of intergroup behavior in the present study, but the results we obtained likely have implications for 
intergroup behaviors. From a theoretical perspective, processes related to the identification and categorization of individuals into social groups is predicted to affect downstream processes, including the activation of beliefs, feelings, and behavioral intentions (Brewer, 1988; Fiske \& Neuberg, 1990). Moreover, because attentional processes affect categorization (Medin \& Schaffer, 1978; Smith \& Zarate, 1992), categorybased differences in attention should affect intergroup behavior. Consistent with this, we have recently shown that individual differences in attention to ingroup and outgroup members predict implicit evaluative bias (Ito, WilladsenJensen, \& Park, 2007). This study used an evaluative priming task in which Black and White face primes appeared before positive and negative words (cf. Fazio, Jackson, Dunton, \& Williams, 1995). Individuals who showed larger differences in early attentional ERP responses to ingroup relative to outgroup primes also showed greater ingroup evaluative bias in their pattern of response latencies to the word stimuli.

Participants in Ito et al. (2007) were passively viewing the face primes. Thus, the individual variability in ERP responses reflects spontaneous differences in how people direct attention as a function of race, and shows that these differences contribute to the evaluative associations that are activated by those faces. We would expect the attentional differences observed in the present study to similarly relate to evaluative associations. Of interest, Ito et al. used a primarily White sample, and did not examine the role of contextual influences. If non-White participants are more sensitive to racial context, it would be particularly interesting to determine whether the influence of context on the early perceptual responses has concomitant effects on subsequent evaluative associations. Moreover, relations between the ERP and implicit evaluative bias effects were most consistently obtained in the N200. In line with the earlier discussion that ERPs afford an opportunity to selectively assess individual aspects of racial perception, these results suggest that individual differences related to depth of processing may play a bigger role in the activation of evaluative associations as compared to other processes, such as vigilance. These questions have not yet been addressed, but the ability to assess them highlights the potential contribution of neuroscience measures in general and ERPs in particular to the study of intergroup behavior.

\section{Notes}

1. In past research, we have also referred to the LPP as the P300. P300 is generally considered to be a more specific name, referring to a positivegoing component that tends to peak around $300 \mathrm{~ms}$, and is sensitive to incongruities between the present stimulus and stimuli that precede it. LPP is a more general term referring to a positive-going potential that occurs relatively late in processing (e.g. several hundred ms after stimulus onset), and may be obtained in response to a range of manipulations. Although the potential we quantify in this study was elicited in a paradigm in which we manipulated the similarity between the present and preceding stimuli, we choose to use the more general LPP name in part to avoid confusion given the presence of the N400 component, which had a shorter latency than our LPP/P300.

2. The absence of race effects in the N100 replicates previous research (Willadsen-Jensen \& Ito, 2006).

3. Because the scalp site factor did not interact in a theoretically important way with other factors, any significant effects that involved scalp site are footnoted.

4. A scalp site main effect emerged in the P200 analysis: $F(2,38)=14.53, p<.001$. P200s were larger at $\mathrm{Pz}(M=5.56 \mu \mathrm{V})$ than $\mathrm{Cz}(M=4.25 \mu \mathrm{V})$ or $\mathrm{Fz}(M=3.23 \mu \mathrm{V}),\left(F_{\mathrm{S}}(1,19)=18.23\right.$ and 7.63 , respectively, $p \mathrm{~s}<.05)$. P200s were also significantly larger at $\mathrm{Cz}$ than $\mathrm{Fz},(F(1,19)=6.56$, $p<.05)$. Following Jennings (1987), the sagittal effects were evaluated using a GreenhouseGeisser correction, although uncorrected degrees of freedom are reported for ease.

5. The N200 analysis also showed a significant sagittal main effect $(F(2,38)=37.52, p<.0001)$. $\mathrm{N} 200$ s were significantly larger at $\mathrm{Fz}(M=-3.29 \mu \mathrm{V})$ and $\mathrm{Cz}(M=-2.91 \mu \mathrm{V})$ than $\mathrm{Pz}(F \mathrm{~s}(1,19)=44.63$ and $69.49, p \mathrm{~s}<.0001)$, respectively. N200s did not differ at $\mathrm{Fz}$ and $\mathrm{Cz}(F(1,19)=1.38, \mathrm{~ns})$. 
6. In our past research with White participants (Willadsen-Jensen \& Ito, 2006), results in the N400 were identical to those of the subsequently occurring LPP/P300.

7. There was also a theoretically uninformative significant main effect of Target Race $(F(2,38)$ $=4.95, p<.05)$. LPPs were larger to the racially ambiguous faces $(M=8.60 \mu \mathrm{V})$ as compared to White $(M=7.02 \mu \mathrm{V})$ and Asian faces $(M=7.16 \mu \mathrm{V}),\left(F_{\mathrm{s}}(1,19)=6.02\right.$ and 6.35 , $p s<.05$, respectively). Because the LPP is affected by context, this target race main effect simply reflects the fact that faces were seen in the context of White and Asian faces, which resulted in larger average amplitudes to targets that never appeared as the frequently presented context (i.e. the racially ambiguous faces). The sagittal main effect, $(F(2,38)=49.23, p<.0001)$ revealed that $\mathrm{P} 300 \mathrm{~s}$ were larger at $\mathrm{Pz}(M=10.33 \mu \mathrm{V})$ than $\mathrm{Cz}(M=7.64 \mu \mathrm{V})$, and at $\mathrm{Cz}$ than $\mathrm{Fz}(M=4.82 \mu \mathrm{V}),\left(F_{\mathrm{S}}(1,19)=32.82\right.$ and $35.56, p \mathrm{~s}<.0001)$.

8. For three of the participants, explicit categorization data were not recorded because they did not follow the instructions to indicate their responses on the keypad. Their data were included in the ERP analyses, but not in the categorization analyses.

\section{Acknowledgement}

This research was supported by National Institute of Mental Health grant R01 MH071257 to Tiffany A. Ito. We are grateful to the CU Stereotyping and Prejudice lab and Jordan Wood for comments on earlier versions of this work.

\section{References}

Anthony, T., Copper, C., \& Mullen, B. (1992). Cross-racial facial identification: A social cognitive integration. Personality and Social Psychology Bulletin, 18, 296-301.

Bar-Haim, Y., Ziv, T., Lamy, D., \& Hodes, R. M. (2006). Nature and nurture in own-race face processing. Psychological Science, 17, 159-163.

Bentin, S., \& Deouell, L.Y. (2000). Structural encoding and identification in face processing: ERP evidence for separate mechanisms. Cognitive Neuropsychology, 17, 35-54.

Bodenhausen, G. V., \& Macrae, C. N. (1998). Stereotype activation and inhibition. Advances in Social Cognition, 11, 1-52.
Brewer, M. B. (1988). A dual process model of impression formation. Advances in Social Cognition, 1, 1-36.

Buckhout, R., \& Regan, S. (1988). Explorations in research on the other-race effect in face recognition. In M. M. Gruneberg, P. E. Morris, \& R. N. Sykes (Eds.), Practical aspects of memory: Current research and issues: Vol. 1. Memory in everyday life (pp. 40-46). Oxford, UK: Wiley.

Cacioppo, J. T., Crites, S. L., Jr., Berntson, G. G., \& Coles, M. G. H. (1993). If attitudes affect how stimuli are processed, should they not affect the event-related brain potential? Psychological Science, 4, 108-112.

Chance, J. E., \& Goldstein, A. G. (1981). Depth of processing in response to own- and other-race faces. Personality and Social Psychology Bulletin, 7, $475-480$.

Chiroro, P., \& Valentine, T. (1995). An investigation of the contact hypothesis of the own-race bias in face recognition. Quarterly Journal of Experimental Psychology, 48A, 879-894.

Corneille, O., Huart, J., Becquart, E., \& Brédart, S. (2004) When memory shifts toward more typical category exemplars: Accentuation effects in the recollection of ethnically ambiguous faces. Journal of Personality and Social Psychology, 86, 236-250.

Dickter, C. L., \& Bartholow, B. D. (2007). Racial ingroup and outgroup attention biases revealed by event-related brain potentials. Social, Cognitive, and Affective Neuroscience, 2, 189-198.

Donchin, E. (1981). Surprise!...Surprise? Psychophysiology, 18, 493-513.

Eberhardt, J., Dasgupta, N., \& Banaszynski, T. L. (2003). Believing is seeing: The effects of racial labels and implicit beliefs on face perception. Personality and Social Psychology Bulletin, 29, 360-370.

Eimer, M. (2000). Event-related brain potentials distinguish recognition. Clinical Neurophysiology, 111, 694-705.

Eimer, M., \& Holmes, A. (2002). An ERP study on the time course of emotional face processing. NeuroReport, 13, 427-431.

Eimer, M., \& Holmes, A., \& McGlone, F. (2003). The role of spatial attention in the processing of facial expression: An ERP study of rapid brain responses to six basic emotions. Cognitive, Affective, and Behavioral Neuroscience, 3, 97-110.

Fazio, R. H., Jackson, J. R., Dunton, B. C., \& Williams, C. J. (1995). Variability in automatic activation as an unobtrusive measure of racial 
attitudes: A bona fide pipeline? Journal of Personality and Social Psychology, 69, 1013-1027.

Feinman, S., \& Entwisle, D. R. (1976). Children's ability to recognize other children's faces. Child Development, 47, 506-510.

Fiske, S. T., \& Neuberg, S. L. (1990). A continuum of impression formation, from category - based to individuating processes: Influences of information and motivation on attention and interpretation. Advances in Experimental Social Psychology, 23, 1-73.

Golby, A.J., Gabrieli, J.D.E., Chiao, J.Y., \& Eberhardt, J.L. (2001). Differential responses in the fusiform region to same-race and other-race faces. Nature Neuroscience, 4, 845-850.

Goldstein, A. G., \& Chance, J. E. (1980). Memory for faces and schema theory. Journal of Psychology, 105, 47-59.

Hillyard, S. A., \& Munte, T.F. (1984). Selective attention to color and location: An analysis with event-related brain potentials. Perception and Psychophysics, 36, 185-198.

Ito, T. A., Larsen, J. T., Smith, N. K., \& Cacioppo, J. T. (1998). Negative information weighs more heavily on the brain: The negativity bias in evaluative categorizations. Journal of Personality and Social Psychology, 75, 887-900.

Ito, T. A., Thompson, E., \& Cacioppo, J. T. (2004). Tracking the timecourse of social perception: The effects of racial cues on event-related brain potentials. Personality and Social Psychology Bulletin, 30, 1267-1280.

Ito, T. A., \& Urland, G. R. (2003). Race and gender on the brain: Electrocortical measures of attention to race and gender of multiply categorizable individuals. Journal of Personality and Social Psychology, 85, 616-626.

Ito, T. A., \& Urland, G. R. (2005). The influence of processing objectives on the perception of faces: An ERP study of race and gender perception. Cognitive, Affective, and Behavioral Neuroscience, 5, 21-36.

Ito, T.A., Willadsen-Jensen, E. C., \& Park, P. (2007). From perception to evaluation: Early attention and automatic activation of bias to racially ambiguous faces. Manuscript under review.

Jennings, J.R. (1987). Editorial policy on analyses of variance with repeated measures. Psychophysiology, 24, 474-478.

Karylowski, J. J., Motes, M. A., Curry, D., \& Van Liempd, D. (2002). 'In what font color is Bill Cosby's name written?': Automatic racial categorization in a Stroop task. North American Journal of Psychology, 4, 1-12.
Kubota, J. T., \& Ito, T. A. (2007). Multiple cues in social perception: The time course of processing race and facial expression. Journal of Experimental Social Psychology, 43, 738-752.

Levin, D. T. (1996). Classification of faces by race: The structure of face categories. Journal of Experimental Psychology: Learning, Memory, and Cognition, 22, 1364-1382.

Levin, D. T. (2000). Race as a visual feature: Using visual search and perceptual discrimination tasks to understand face categories and the crossrace recognition deficit. Journal of Experimental Psychology: General, 129, 559-574.

Luck. S. J., \& Hillyard, S. A. (1994). Electrophysiological correlates of feature analysis during visual search. Psychophysiology, 31, 291-308.

MacLin, O. H., \& Malpass, R. S. (2001). Racial categorization of faces: The ambiguous race face effect. Psychology, Public Policy and Law, 7, 98-118.

Malpass, R. S., \& Kravitz, J. (1969). Recognition for faces of own and other race. Journal of Personality and Social Psychology, 13, 330-334.

Medin, D. L., \& Schaffer, M. M. (1978). Context theory of classification learning. Psychological Review, 85, 207-238.

Ng, W.-J, \& Lindsay, R. C. L. (1994). Cross-race facial recognition: Failure of the contact hypothesis. Journal of Cross-Cultural Psychology. 25, 217-232.

Pezdek, K., Blandon-Gitlin, I., \& Moore, C. (2003). Children's face recognition memory: More evidence for the cross-race effect. Journal of Applied Psychology, 88, 760-763.

Rhodes, G., Brennan, S., \& Carey, S. (1987). Identification and ratings of caricatures: Implications for mental representations of faces. Cognitive Psychology, 19, 473-497.

Ritter, W., Simson, R., \& Vaughan, H. G. (1983). Event-related potential correlates of two stages of information processing in physical and semantic discrimination trials. Psychophysiology, 20, 168-179.

Sangrigoli, S., \& de Schonen, S. (2004). Effect of visual experience on face processing: A developmental study of inversion and nonnative effects. Developmental Science, 7, 74-87.

Sangrigoli, S., Pallier, C., Argenti, A. M., Ventureyra, V.A.G., \& de Schonen, S. (2005). Reversibility of the other-race effect in face recognition during childhood. Psychological Science, 16, 440-444.

Sekaquaptewa, D., Waldman, A., \& Thompson, M. (2007). Solo status and self-construal: Being 
distinctive influences racial self-construal and performance apprehension in African American women. Cultural Diversity and Ethnic Minority Psychology, 13, 321-327.

Semlitsch, H. V., Anderer, P., Schuster, P., \& Presslich, O. (1986). A solution for reliable and valid reduction of ocular artifacts, applied to the LPP ERP. Psychophysiology, 23, 695-703.

Shapiro, P. N., \& Penrod, S. (1986). Meta-analysis of facial identification studies. Psychological Bulletin, 100, 139-156.

Smith, E. R., \& Zarate, M. A. (1992). Exemplarbased model of social judgment. Psychological Review, 99, 3-21.

Stangor, C., Lynch, L. Duan, C., \& Glass, B. (1992). Categorization of individuals on the basis of multiple social features. Journal of Personality and Social Psychology, 62, 207-218.

Tanaka, J. W, Curran, T., Porterfield, A. L., \& Collins, D. (2006). Activation of preexisting and acquired face representations: The N250 eventrelated potential as an index of face familiarity. Journal of Cognitive Neuroscience, 18, 1488-1497.

Valentine, T. (1991) A unified account of the effects of distinctiveness, inversion, and race in face recognition. Quarterly Journal of Experimental Psychology, 43A, 161-204.

Valentine, T., \& Bruce, V. (1986). The effect of race, inversion and encoding activity upon face recognition. Acta Psychologica, 61, 259-273.
Valentine, T., \& Endo, M. (1992). Towards an exemplar model of face processing: The effects of race and distinctiveness. Quarterly Journal of Experimental Psychology, 44A, 671-703.

Wijers, A. A., Mulder, G., Okita, T., Mulder, L. J. M., \& Scheffers, M. K. (1989). Attention to color: An analysis of selection, controlled search, and motor activation, using event-related potentials. Psychophysiology, 26, 89-109.

Willadsen-Jensen, E. C., \& Ito, T. A. (2006). Ambiguity and the timecourse of racial categorization. Social Cognition, 24, 580-606.

\section{Biographical notes}

EVE C. Willadsen-JENSEN (PhD, University of Colorado-Boulder) is a research consultant at Target Corporation. Her research interests include group processes, racial categorization, stereotyping and prejudice, and workplace diversity.

TIFfany a. ITo received her PhD from the University of Southern California in 1990 and is currently an associate professor at the University of Colorado. Her research integrates neuroscience theories and methods in the study of social perception, especially focusing on issues related to social categorization, stereotyping, and prejudice. 